\title{
Fulminant Essential Thrombocythemia Associated with Acquired Von Willebrand Syndrome and Bleeding Episodes in a 14-year- old Girl
}
C. Schneider ${ }^{1}$
E. Stutz-Grunder ${ }^{1}$
S. Lüer ${ }^{1}$
P. Keller ${ }^{2}$
J. A. Kremer Hovinga ${ }^{2}$
R. A. Ammann ${ }^{1}$
A. Karow ${ }^{1}$

${ }^{1}$ Division of Pediatric Hematology/Oncology, Department of Pediatrics, Inselspital, Bern University Hospital, University of Bern, Bern, Switzerland

2 Department of Hematology, University Hospital/Inselspital Bern and University of Bern, Bern, Switzerland

\begin{abstract}
Address for correspondence C. Schneider, Dr. med., Division of Pediatric Hematology/Oncology, Department of Pediatrics, Inselspital, Bern University Hospital, University of Bern, Bern, Switzerland (e-mail: christine.schneider@insel.ch).
\end{abstract}

Hämostaseologie 2019;39:404-408.

\begin{abstract}
Keywords

- essential thrombocythemia

- myeloproliferative neoplasm

- Von Willebrand syndrome

Background Essential thrombocythemia is a chronic myeloproliferative neoplasm. It is extremely rare in children below 15 years of age with an estimated annual incidence of only 0.09 per million. Usually, clinical symptoms associated with essential thrombocythemia are mild or absent.

Case Here, we present the case of a 14-year-old female patient fulminantly presenting with acute symptoms comprising visual impairment, palmar and plantar stabbing pain. Blood count revealed massive thrombocytosis of $2373 \times 10^{9} / \mathrm{L}$. Bone marrow morphology showed elevated numbers of mature megakaryocytes. Von Willebrand factor activity/antigen ratio was significantly reduced compatible with an acquired Von Willebrand syndrome associated with high platelet counts. Molecular analyses for driver mutations of myeloproliferative neoplasms including JAK2V617F, CALR and MPL were negative. Acute therapy comprising hyperhydration and oxygen supply complemented by acetylsalicylic acid led to amelioration of symptoms. Medication with hydroxycarbamide maintained a significant reduction of platelet counts but had to be reduced or withheld several times due to neutropenia. Repeated bleeding episodes observed in the course were clearly associated with increases in platelet counts above $1200 \times 10^{9} / \mathrm{L}$ explained by acquired von Willebrand syndrome. Sixteen months after diagnosis, therapy was switched to pegylated interferon and platelet counts could be stabilized without significant side effects.
\end{abstract}

\section{Introduction}

Essential thrombocythemia (ET) is a chronic myeloproliferative neoplasm (MPN) with clonal megakaryocyte proliferation. It is an extremely rare cause of thrombocytosis in childhood and adolescence with an estimated annual inci- dence of 0.09 per million under 15 year olds. ${ }^{1}$ Especially in the young symptoms of ET are mild or even absent and the disease is often diagnosed only accidentally. Therefore, real incidence of ET is likely to be underestimated in these patients. In contrast to ET in adults thromboembolic events or bleeding complications are uncommon in children ${ }^{2}$ and received

September 4, 2018

accepted after revision

January 17, 2019 (c) 2019 Georg Thieme Verlag KG Stuttgart · New York
DOI https://doi.org/ $10.1055 / \mathrm{s}-0039-1679929$. ISSN 0720-9355. 
somatic mutations are rarely present in childhood ET. ${ }^{3}$ Therefore, diagnosis of childhood ET is challenging and World Health Organization (WHO) diagnostic criteria for adult MPN cannot be simply adopted. ${ }^{2,4}$ In general, ET is associated with a good prognosis, ${ }^{5}$ but life expectancy is limited when thromboembolic complications or progression to post-ET-myelofibrosis or acute myeloid leukaemia (AML) occurs. Here, we report on a case of a 14-year-old girl with an impressively fulminant clinical presentation of ET. Symptoms were highly acute and comprised headache, visual impairment and painful paresthesia of the upper and lower extremities. Extraordinarily high platelet counts were found and urgent intervention was required.

\section{Case Report}

A 14-year-old female patient consulted her general practitioner with recurrent stabbing pain and palmoplantar erythema since 2 weeks. Blood count performed to exclude underlying infection revealed massive thrombocytosis above the diagnostic range of the analyser used and the girl was referred to the emergency department of our university hospital. Two weeks before, when the patient consulted her general practitioner because of first mild pain episodes, blood count had been performed for the same reason and showed platelets of $1310 \times 10^{9} / \mathrm{L}$. Retrospectively, elevated platelet counts of more than $600 \times 10^{9} / \mathrm{L}$ for the past 3 years and $996 \times 10^{9} / \mathrm{L} 12$ months before diagnosis had been documented, measured in blood counts examined for suspicion of iron- and vitamin D deficiency and fever. These results had not led to further investigations.

Further history revealed paresthesia of both arms and intermittent headache with impaired vision. Vital signs and clinical findings, including a detailed neurological examination, were normal. In particular, there were no signs of secondary causes for thrombocytosis, i.e. infection, trauma or recent surgery.

Apart from vitamin D substitution there was no other medication nor any drug abuse. The adolescent had so far been healthy. The patient's parents were not consanguineous and had immigrated from Afghanistan when the patient was 9 years old. Apart from multiple miscarriages on the father's side of the family, family history was unremarkable with no thromboembolic events or bleeding complications. Platelet counts of all first-degree relatives were below $300 \times 10^{9} / \mathrm{L}$.

At our institution, the complete blood count was repeated and confirmed thrombocytosis of $2373 \times 10^{9} /$ L determined by a Sysmex XN 9000 platelet counter. Haemoglobin and a differential leukocyte count were within age-specific range.

Laboratory testing showed a level of C-reactive protein $<3$ $\mathrm{mg} / \mathrm{L}$. Serologic testing for Epstein-Barr virus, cytomegalovirus, hepatitis $A, B, C$ and human immunodeficiency virus was negative. Further diagnostics showed a prolonged partial thromboplastin time of 45 seconds (normal range: 2536 seconds). Because of the known relationship of very high platelet counts $\left(>1000 \times 10^{9} / \mathrm{L}\right)$ and acquired von Willebrand syndrome, corresponding laboratory analysis was performed as an explanation for the prolonged partial thromboplastin time. Other reasons for the prolonged partial thromboplastin time were not found. No use of oral anticoagulants or heparin was reported. Factor VIII plasma activity (95\%) was measured later in the course. Lupus anticoagulant and anti-cardiolipin antibody titre were not determined.

Both von Willebrand factor (VWF) activity (27\%) and VWF antigen (48\%) were reduced with a pathological VWF activity/antigen ratio of 0.56 (normal $\geq 0.7$ ). Multimer analysis of VWF revealed a loss of the largest and relative reduction of large VWF multimers as well as accentuated satellite bands (-Fig. 1, lane 1).

Ultrasound showed slight splenomegaly. Bone marrow histology demonstrated increased numbers of mature megakaryocytes exhibiting “staghorn' nuclei. In addition, mild fibrosis without typical features of primary myelofibrosis (increased reticulin or collagen fibres, hyperchromatic or ‘cloud-like' nuclei) was seen (-Fig. 2).

Based on these classical findings, we diagnosed ET. However, histologically, there is no clear distinction between a congenital thrombocytosis and ET and also a 'prefibrotic' primary myelofibrosis could not be entirely excluded.

Screening for the most common somatic mutations known to be associated with MPN using clinical routine assays was negative for JAK2V617F, MPL W515L/K and deletions in calreticulin, respectively. Translocation of BCR-ABL1 was also ruled out. In addition, we analysed the mutational

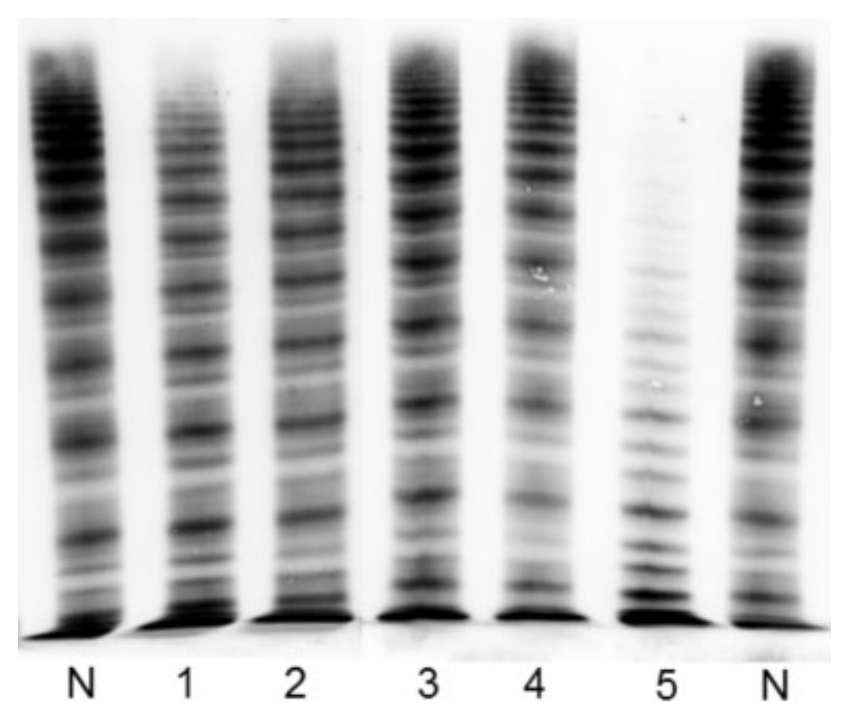

Fig. 1 Von Willebrand factor (VWF) multimer analyses. Separation of von Willebrand factor multimers in a medium resolution $(1.7 \%$ agarose) gel. Lanes N: normal human plasma pool; patient's samples (lanes 1-4): lane 1: at diagnosis (platelet count: $2505 \times 10^{9} / \mathrm{L}$; VWF ratio: 0.45 ; the loss of the large VWF multimers is clearly visible, satellite bands are slightly more prominent than in normal human plasma pool); lane 2: 2 months later (platelet count: $1243 \times 10^{9} / \mathrm{L}$; VWF ratio: 0.65 ); lane 3: 10 months after diagnosis (platelet count: $688 \times 10^{9} / \mathrm{L}$; VWF ratio: 0.79$)$; lane 4: 11 months after diagnosis (platelet count: $267 \times 10^{9} / \mathrm{L}$; VWF ratio: 0.88 ); lane 5: plasma of a patient with genetically confirmed Von Willebrand disease 2A (IIA) (VWF ratio: 0.25 ) characterized by loss of large and medium sized VWF multimers and clearly accentuated satellite bands. 


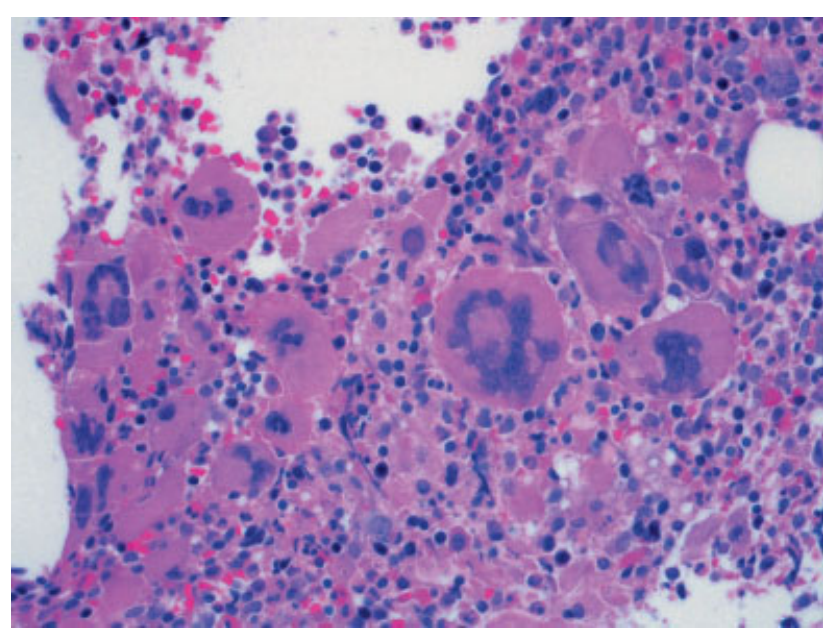

Fig. 2 Bone marrow histology. May-Grünwald-Giemsa ( $\times 400$ magnification). Initial bone marrow histology showed mature megakaryocytes with a lobulated nucleus also known as 'staghorn' nuclei typically seen in essential thrombocythemia. No evidence of increased reticulin or collagen fibres, hyperchromatic or 'cloud-like' nuclei. The remaining cell lines were normal.

spectrum in our patient using a targeted next-generation sequencing panel comprising all exons and the flanking regions of JAK2, MPL and TPO and more than 60 cancerrelated genes. However, even this approach did not detect any clonal aberration.

Because of massive thrombocytosis with associated neurological symptoms, treatment with hydration (2-3 L daily) and oxygen supply was initiated and oral acetylsalicylic acid (100 mg increased to $200 \mathrm{mg}$ divided in two daily doses) was started. Cerebral ischaemia was excluded by an emergency magnetic resonance imaging (MRI) scan.

A therapy with oral $20 \mathrm{mg} / \mathrm{kg}$ hydroxycarbamide (Litalir) daily was started and gradually increased to $40 \mathrm{mg} / \mathrm{kg}$ daily.
Due to persisting symptomatic thrombocytosis 2 weeks after initiation of treatment, platelet apheresis was performed. Since the patient's initial ferritin of $34 \mu \mathrm{g}$ (range: $7-140 \mu \mathrm{g}$ ) was always in a low normal range with borderline haemoglobin levels of $120 \mathrm{~g} / \mathrm{L}$ (range: 121-154 g/L), we decided to repeatedly apply iron sucrose to minimize any possible additional reactive thrombocytosis due to effective iron deficiency. However, we observed no effect on platelet counts.

Because of neutropenia, hydroxycarbamide had to be reduced twice and stopped three times for several days, respectively. As a consequence, platelet counts repeatedly increased significantly at around 3 weeks after stopping hydroxycarbamide (-Fig. 3).

Due to these fluctuations of cell counts and the convincing experience with pegylated interferon in adult patients, we replaced hydroxycarbamide 16 months after the first presentation and switched to treatment with peginterferon alfa2a (Pegasys) as a subcutaneous injection starting with 90 $\mathrm{mcg} /$ week and continuous increase of the dose up to 180 $\mathrm{mcg} /$ week over 3 months.

Under this treatment, the patient remained asymptomatic, platelet counts appeared stabilized at around $800 \times 10^{9} / \mathrm{L}$ and no side effects were observed.

Persistent reduction of the platelet count also led to normalization of the VWF ratio.

Notably, there were three episodes with increased bleeding tendency in the further course, two with epistaxis and one with significantly prolonged menstrual bleeding. All of these episodes were associated with an increase in platelet counts (-Fig. 3 ).

Follow-up bone marrow biopsy taken 1 year after diagnosis showed stable histologic features.

-Fig. 3 illustrates the clinical course and platelet count over time with respect to different therapeutic interventions.

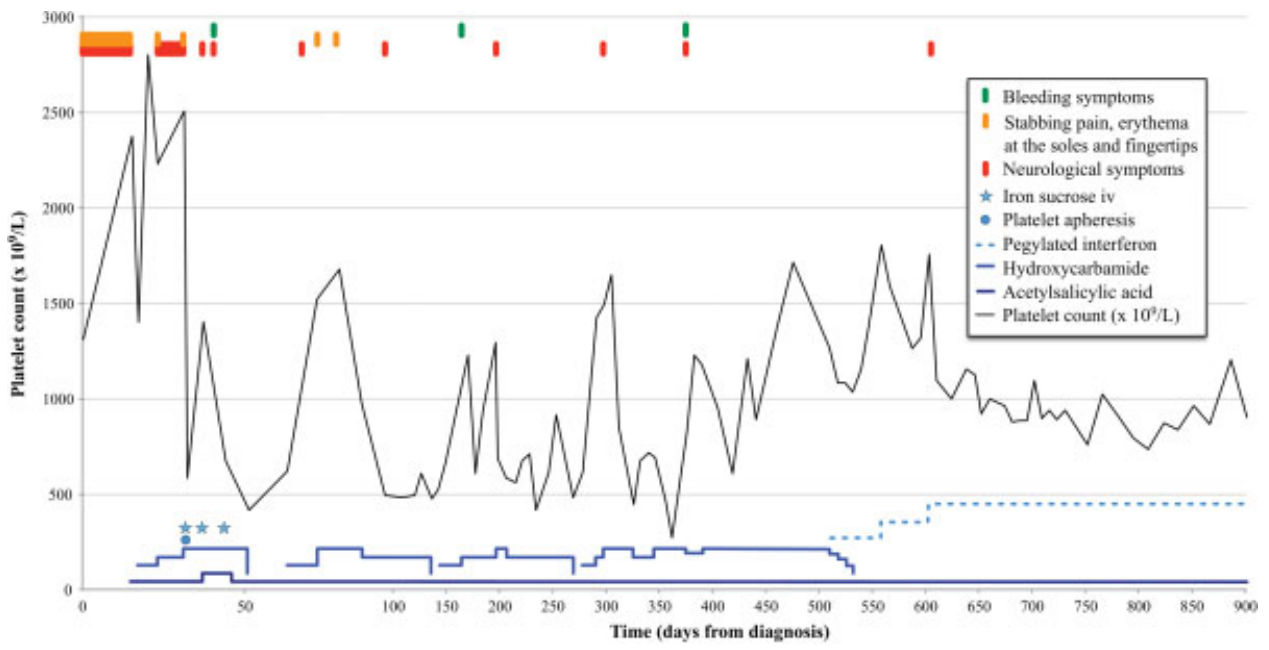

Fig. 3 Clinical symptoms, laboratory findings and therapeutic course. Measured along the horizontal axis is a follow-up of 30 months as days from diagnosis. The vertical scale represents platelet count. Observations showed platelet count above $700 \times 10^{9} / \mathrm{L}$ caused bleeding and neurological symptoms, stabbing pain and erythema at the soles and fingertips. Two weeks after diagnosis, platelet apheresis was performed and showed transient decrease in platelet count. Intravenous iron sucrose showed no impact on platelet count. The dose-dependent effect of treatment with hydroxycarbamide $(20 \mathrm{mg} / \mathrm{kg}$ increased to $40 \mathrm{mg} / \mathrm{kg}$ ) on platelet counts is also illustrated. 


\section{Discussion}

The most common cause of elevated platelet counts in adolescence is reactive, mostly infection-associated, thrombocytosis. These cases are self-limiting and remain asymptomatic. Complications like bleeding or thrombosis are hardly seen in reactive thrombocytosis. ${ }^{6,7}$

Other reasons for reactive thrombocytosis are surgery, anaemia in particular due to iron deficiency, drug side effects, allergy, ${ }^{7,8}$ chronic inflammatory disease and asplenia. $^{9}$

One further but rare differential diagnosis is congenital thrombocytosis, an inherited autosomal-dominant, autosomal-recessive or X-linked condition. However, in our case all family members of the first generation had platelet counts below $300 \times 10^{9} / \mathrm{L}$ and previous blood samples from our patient taken by the family physician showed lower platelet values gradually increasing over months. These findings and on the absence of mutations in TPO and MPL as shown in our diagnostic screening rendered the diagnosis of congenital thrombocytosis unlikely and we diagnosed ET.

ET is an extremely rare cause of thrombocytosis in adolescence. According to the current WHO criteria, diagnosis of ET is made when the following criteria ${ }^{4}$ are fulfilled: (1) prolonged platelet count over $450 \times 10^{9} / \mathrm{L}$, (2) bone marrow aspiration showing an elevated number of mature megakaryocytes, (3) not matching one of the WHO criteria for other chronic Philadelphia-negative myeloid diseases and (4) confirmation of one acquired somatic mutation or clonal marker. If the last criterion is not met, the diagnosis of ET can be made after 1 year without evidence of reactive thrombocytosis. In our patient, no somatic mutation or clonal marker was identified while all other criteria were present. Gene mutations typically found in adult MPN are often lacking in children and adolescents suggesting a different disease pathology. ${ }^{10}$

Only very few paediatric patients with ET present with microcirculatory symptoms, thromboembolic events, haemorrhagic diathesis or splenomegaly. Symptoms in our patient were uncommonly severe and together with extremely high platelet numbers prompted immediate initiation of treatment.

Patients with ET have an elevated risk of thrombotic events but also may show a bleeding tendency. The latter is explained by the quantitative and qualitative platelet alterations resulting in thrombocytopathy which is, as in our case, often aggravated by an acquired Von Willebrand syndrome, the result of adsorption of large VWF multimers on platelets. ${ }^{11-13}$ Platelets with increased degradation might explain the overproportional reduction of VWF activity with a decreased activity/antigen ratio. These mechanisms have likely caused the episodes of increased bleeding tendency with epistaxis and prolonged menstrual bleeding observed in our patient. It cannot be ruled out that the medication with aspirin augmented this bleeding tendency. The initial therapeutic decision to apply aspirin, however, was based on the significantly increased risk of thromboembolic complications associated with the extremely high platelet counts found in our patient with ET. The two bleeding episodes observed in our patient were clinically not severe. After these minor bleeding episodes, we intermittently halved the aspirin dosage. Since bleeding symptoms occurred in phases of increased platelet counts $>1200 \times 10^{9} / \mathrm{L}$ and subsided when platelet counts dropped, it can be assumed that they were mainly caused by the secondary Von Willebrand syndrome.

Regarding the low numbers of treated children with ET and the long-term implications in this age group, the therapeutic approach of childhood and adolescence MPN remains challenging.

We have chosen to use hydroxycarbamide as primary cytoreductive therapy in our patient because of our experience with this medication in sickle cell anaemia patients where hydroxycarbamide is used for the induction of foetal haemoglobin and acts as an endothelial cell adhesion modulator. ${ }^{14}$ However, an aggravated leukaemogenic potential in adult ET patients after long-term use is still controversially debated. In addition, due to its potential fetotoxic and embryotoxic effects, hydroxycarbamide will have to be replaced for pregnancy.

Alternative therapeutic options are anagrelide and pegylated interferon (Pegasys). Both substances are established in the treatment of adult MPN. ${ }^{15}$

Like polycythemia vera, ET can transform to myelofibrosis or AML. No prognostic markers are known to predict transformation. Due to the mild bone marrow fibrosis observed in our patient however, we could not entirely exclude 'prefibrotic' primary myelofibrosis which is associated with a less optimal prognosis.

In summary, diagnosis of ET is very rare in children and adolescents and should be considered as a differential diagnosis in persistent thrombocytosis only after exclusion of secondary causes. Childhood ET appears to have a different genetic background than adult ET and often mutational status cannot serve as a diagnostic criterion. Paediatric patients with ET are mostly asymptomatic and only a very small minority present with microcirculatory symptoms, thromboembolic events, haemorrhagic diathesis or splenomegaly. Lacking a standard therapy in children and adolescents, management and especially long-term treatment of ET in these age groups can be challenging.

\section{Conflict of Interest}

The authors have no conflict of interest to disclose.

\section{Acknowledgment}

We thank Emese Györbiro and Yara Banz for expert technical assistance.

\section{References}

$1 \mathrm{Hasle} \mathrm{H}$. Incidence of essential thrombocythaemia in children. $\mathrm{Br} \mathrm{J}$ Haematol 2000;110(03):751

2 Fu R, Zhang L, Yang R. Paediatric essential thrombocythaemia: clinical and molecular features, diagnosis and treatment. $\mathrm{Br} \mathrm{J}$ Haematol 2013;163(03):295-302

3 Karow A, Nienhold R, Lundberg P, et al. Mutational profile of childhood myeloproliferative neoplasms. Leukemia 2015;29(12): 2407-2409 
4 Birgegård G. Advances and challenges in the management of essential thrombocythemia. Ther Adv Hematol 2015;6(03): 142-156

5 Tefferi A, Pardanani A. Myeloproliferative neoplasms: a contemporary review. JAMA Oncol 2015;1(01):97-105

6 Subramaniam N, Mundkur S, Kini P, Bhaskaranand N, Aroor S. Clinicohematological study of thrombocytosis in children. ISRN Hematol 2014;2014:389257

7 Dame C, Sutor AH. Primary and secondary thrombocytosis in childhood. Br J Haematol 2005;129(02):165-177

8 Wang JL, Huang LT, Wu KH, Lin HW, Ho MY, Liu HE. Associations of reactive thrombocytosis with clinical characteristics in pediatric diseases. Pediatr Neonatol 2011;52(05):261-266

9 Kirkineska L, Perifanis V, Vasiliadis T. Functional hyposplenism. Hippokratia 2014;18(01):7-11

10 Lundberg P, Karow A, Nienhold R, et al. Clonal evolution and clinical correlates of somatic mutations in myeloproliferative neoplasms. Blood 2014;123(14):2220-2228
11 Budde U, Scharf RE, Franke P, Hartmann-Budde K, Dent J, Ruggeri ZM. Elevated platelet count as a cause of abnormal von Willebrand factor multimer distribution in plasma. Blood 1993;82(06): 1749-1757

12 Tiede A, Rand JH, Budde U, Ganser A, Federici AB. How I treat the acquired von Willebrand syndrome. Blood 2011;117(25):6777-6785

13 van Genderen PJ, Michiels JJ, van der Poel-van de Luytgaarde SC, van Vliet $\mathrm{HH}$. Acquired von Willebrand disease as a cause of recurrent mucocutaneous bleeding in primary thrombocythemia: relationship with platelet count. Ann Hematol 1994;69(02): 81-84

14 Strouse JJ, Lanzkron S, Beach MC, et al. Hydroxyurea for sickle cell disease: a systematic review for efficacy and toxicity in children. Pediatrics 2008;122(06):1332-1342

15 Gisslinger $\mathrm{H}$, Gotic $\mathrm{M}$, Holowiecki J, et al; ANAHYDRET Study Group. Anagrelide compared with hydroxyurea in WHOclassified essential thrombocythemia: the ANAHYDRET Study, a randomized controlled trial. Blood 2013;121(10):1720-1728 